A 17-year-old boy presented with a 5-day history of melena. His past medical history and his family history were unremarkable. On admission, physical examination revealed pallor and severe hypotension (blood pressure $84 / 42 \mathrm{~mm} \mathrm{Hg}$ ), but no abdominal signs. Hematologic analysis on admission showed a red blood cell count of $101 \times 10^{4} / \mathrm{mm}^{3}$, a hemoglobin level of $2.9 \mathrm{~g} / \mathrm{dl}$ and a hematocrit of $9.2 \%$. Upper gastrointestinal endoscopy demonstrated no bleeding; colonoscopy revealed blood clots but no source of bleeding. Angiography, radio-labeled red-cell scintigraphy, and technetium-99 m pertechnetate scintigraphy also failed to detect the source of bleeding.

Thirteen days after admission, retrograde double-balloon enteroscopy revealed a large diverticulum in the distal part of the ileum and a small ulcer was identified within the diverticulum (Figure 1). The ileal diverticulum, $10 \mathrm{~cm}$ in size, was also seen on barium-contrast radiography of the small intestine (Figure 2 ). A presumptive diagnosis of Meckel's diverticulum was made and the patient underwent laparoscopy. A Meckel's diverticulum measuring $10 \mathrm{~cm}$ in diameter was found approximately $70 \mathrm{~cm}$ proximal to the ileocecal junction (Figure 3), and this was resected laparoscopically. Macroscopically, an ulcer scar was identified in the diverticulum (Figure 4). Histological examination showed ectopic gastric mucosa surrounding the ulcer scar (Figure 5 ). The postoperative course was uneventful and the patient remains in complete remission 6 months after resection of the diverticulum.

Meckel's diverticulum is the most common congenital anomaly of the gastrointestinal tract, with an incidence at autopsy of $0.3 \%-4 \%[1,2]$. The diverticulum is usually situated $40-130 \mathrm{~cm}$ from the ileocecal junction and is therefore difficult to detect endoscopically before surgery [3]. However, double-balloon enteroscopy, developed by Yamamoto et al. [4], enables examination of the entire

\title{
Meckel's diverticulum diagnosed by double-balloon enteroscopy
}
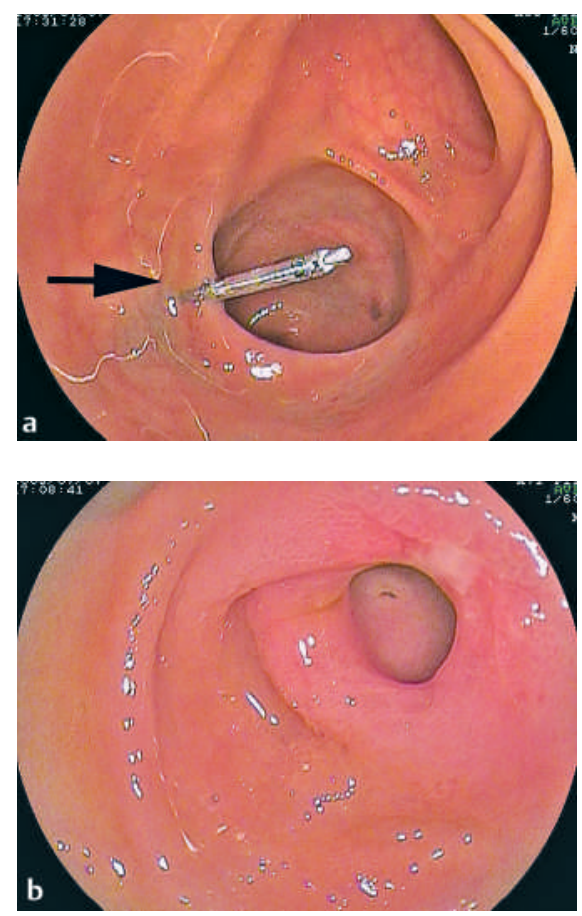

Figure 1 Double-balloon enteroscopic views of the patient's Meckel's diverticulum. The diverticulum can be seen, marked by the arrow, in the inferior part of the ileum (a). A small ulcer and stenosis were seen within the diverticulum (b).

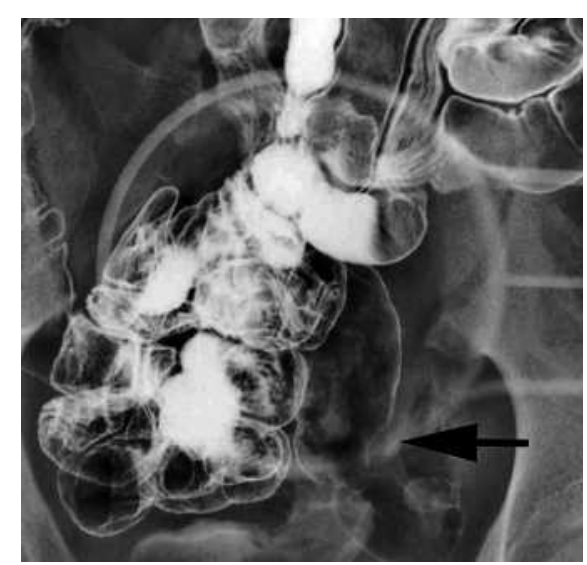

Figure 2 Barium-contrast radiograph of the small intestine showing the diverticulum, approximately $10 \mathrm{~cm}$ in size, in the ileum (arrow).

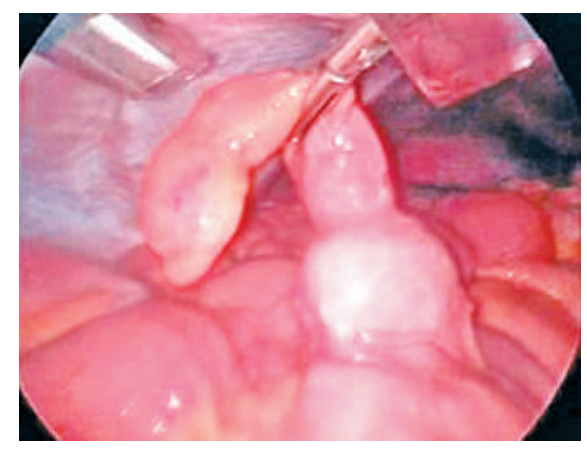

Figure 3 A Meckel's diverticulum measuring $10 \mathrm{~cm}$ was found approximately $70 \mathrm{~cm}$ proximal to the ileocecal junction at laparoscopy.

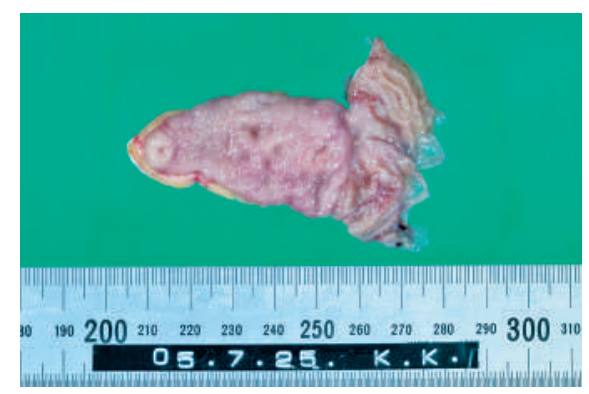

Figure 4 Macroscopic view showing the ulcer scar in the diverticulum.

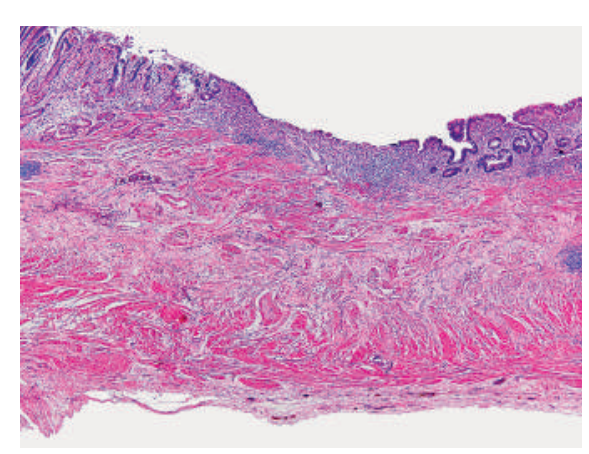

Figure 5 Histological examination revealed ectopic gastric mucosa near the ulcer scar in the diverticulum. 
small intestine, and we were able to visualize this patient's Meckel's diverticulum of the ileum using this procedure. To our knowledge, this is the third report of a Meckel's diverticulum diagnosed preoperatively by double-balloon enteroscopy.

Endoscopy_UCTN_Code_CCL_1AC_2AF

K. Ohnita', H. Isomoto', Y. Mizuta', S. Akazawa', Y. Akazawa', K. Ohba', M. Yoshimura' ${ }^{2}$ S. Shikuwa',

K. Omagari' ${ }^{1}$, F. Fujita ${ }^{3}$, K. Abe ${ }^{4}$, S. Kohno ${ }^{1}$

${ }^{1}$ Second Department of Internal Medicine, Nagasaki University School of Medicine, Nagasaki, Japan

2 Department of Internal Medicine, Nagasaki Yurino Hospital, Nagasaki, Japan

${ }^{3}$ Department of Transplantation and Digestive Surgery, Nagasaki University Graduate School of Biomedical Sciences, Nagasaki, Japan

${ }^{4}$ Department of Pathology, Nagasaki University School of Medicine, Nagasaki, Japan.
References

${ }^{1}$ Aubrey DA. Meckel's diverticulum: a review of the sixty-six emergency Meckel's diverticulectomies. Arch Surg 1970; 100: 144- 146

2 Jay GD, Margulis RR, McGraw AR et al. Meckel's diverticulum; a survey of 109 cases. Arch Surg 1950; 6: 158-169

${ }^{3}$ Weinstein EC, Cain JC, ReMine WH. Meckel's diverticulum: 55 years of clinical and surgical experience. JAMA 1962; 182: 131-133

${ }^{4}$ Yamamoto H, Sekine Y, Sato Y et al. Total enteroscopy with a nonsurgical steerable double-balloon method. Gastrointest Endosc 2001; 53: 216-220
Corresponding author

\section{K. Ohnita, M.D.}

Second Department of Internal Medicine Nagasaki University School of Medicine 1-7-1 Sakamoto

Nagasaki 852-8501

Japan

Fax: $\quad+81-95-849-7568$

Email: k-ohnita@net.nagasaki-u.ac.jp 